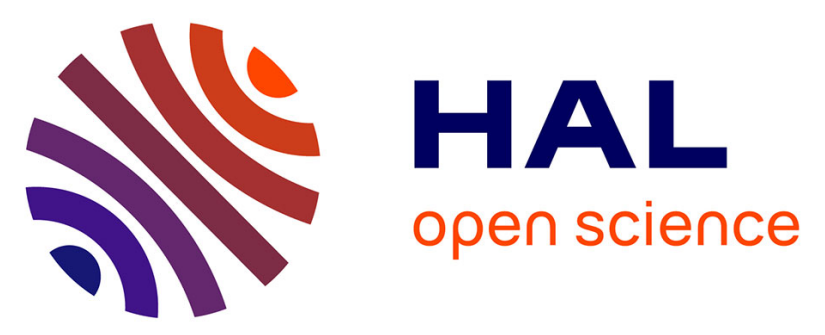

\title{
Immediate interruption of sedation compared with usual sedation care in critically ill postoperative patients (SOS-Ventilation): a randomised, parallel-group clinical trial
}

Gerald Chanques, Matthieu Conseil, Claire Roger, Jean-Michel Constantin, Albert Prades, Julie Carr, Laurent Muller, Boris Jung, Fouad Belafia, Moussa Fafa Cisse, et al.

\section{- To cite this version:}

Gerald Chanques, Matthieu Conseil, Claire Roger, Jean-Michel Constantin, Albert Prades, et al.. Immediate interruption of sedation compared with usual sedation care in critically ill postoperative patients (SOS-Ventilation): a randomised, parallel-group clinical trial. The Lancet Respiratory Medicine, 2017, 5 (10), pp.795-805. 10.1016/s2213-2600(17)30304-1 . hal-01919222

\author{
HAL Id: hal-01919222 \\ https://hal.science/hal-01919222
}

Submitted on 12 Nov 2018

HAL is a multi-disciplinary open access archive for the deposit and dissemination of scientific research documents, whether they are published or not. The documents may come from teaching and research institutions in France or abroad, or from public or private research centers.
L'archive ouverte pluridisciplinaire HAL, est destinée au dépôt et à la diffusion de documents scientifiques de niveau recherche, publiés ou non, émanant des établissements d'enseignement et de recherche français ou étrangers, des laboratoires publics ou privés. 


\section{Immediate interruption of sedation compared with usual sedation care in critically ill postoperative patients (SOS-Ventilation): a randomised, parallel-group clinical trial

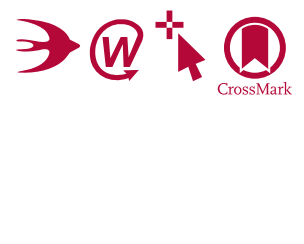

Gerald Chanques, Matthieu Conseil, Claire Roger, Jean-Michel Constantin, Albert Prades, Julie Carr, Laurent Muller, Boris Jung, Fouad Belafia Moussa Cissé, Jean-Marc Delay, Audrey de Jong, Jean-Yves Lefrant, Emmanuel Futier, Grégoire Mercier, Nicolas Molinari, Samir Jaber, on behalf of the SOS-Ventilation study investigators*

\begin{abstract}
Summary
Background Avoidance of excessive sedation and subsequent prolonged mechanical ventilation in intensive care units (ICUs) is recommended, but no data are available for critically ill postoperative patients. We hypothesised that in such patients stopping sedation immediately after admission to the ICU could reduce unnecessary sedation and improve patient outcomes.
\end{abstract}

Methods We did a randomised, parallel-group, clinical trial at three ICUs in France. Stratified randomisation with minimisation (1:1 via a restricted web platform) was used to assign eligible patients (aged $\geq 18$ years, admitted to an ICU after abdominal surgery, and expected to require at least $12 \mathrm{~h}$ of mechanical ventilation because of a critical illness defined by a Sequential Organ Failure Assessment score $>\mathbf{1}$ for any organ, but without severe acute respiratory distress syndrome or brain injury) to usual sedation care provided according to recommended practices (contro group) or to immediate interruption of sedation (intervention group). The primary outcome was the time to successful extubation (defined as the time from randomisation to the time of extubation [or tracheotomy mask] for at least $48 \mathrm{~h}$ ). All patients who underwent randomisation (except for those who were excluded after randomisation) were included in the intention-to-treat analysis. This study is registered with ClinicalTrials.gov, number NCT01486121.

Findings Between Dec 2, 2011, and Feb 27, 2014, 137 patients were randomly assigned to the control (n=68) or intervention groups $(n=69)$. In the intention-to-treat analysis, time to successful extubation was significantly lower in the intervention group than in the control group (median $8 \mathrm{~h}$ [IQR 4-36] $v s 50 \mathrm{~h}$ [29-93], group difference $-33.6 \mathrm{~h}$ $[95 \% \mathrm{CI}-44 \cdot 9$ to $-22 \cdot 4]$; $\mathrm{p}<0 \cdot 0001)$. The adjusted hazard ratio was $5 \cdot 2(95 \% \mathrm{CI} 3 \cdot 1-8 \cdot 8, \mathrm{p}<0 \cdot 0001)$.

Interpretation Immediate interruption of sedation in critically ill postoperative patients with organ dysfunction who were admitted to the ICU after abdominal surgery improved outcomes compared with usual sedation care. These findings support interruption of sedation in these patients following transfer from the operating room.

Funding Délégation à la Recherche Clinique et à l'Innovation du Groupement de Coopération Sanitaire de la Mission d'Enseignement, de Recherche, de Référence et d'Innovation (DRCI-GCS-MERRI) de Montpellier-Nîmes.

\section{Introduction}

More than 300 million surgical procedures are done worldwide each year. ${ }^{1}$ When organ dysfunction occurs during surgery (eg, haemorrhagic or septic shock, or acute respiratory failure), patients are directly admitted to intensive care units (ICUs) for organ support, including mechanical ventilation and sedation. ${ }^{2}$ However, general anaesthesia and sedation in the ICU could distort the perceived severity of a patient's condition because sedatives have complex interactions with organ dysfunction, reduce respiratory drive and blood pressure, and therefore increase the need for mechanical ventilation and vasopressors. Moreover, an abundant literature, mostly concerning medical ICU patients, has shown that sedation is associated with increased morbidity, resource utilisation, and costs because of delayed ventilator weaning, prolonged ICU and hospital stays, and complications (especially delirium). ${ }^{3-6}$ Avoidance of unnecessary deep or prolonged sedation is now a key objective when caring for critically ill patients., Worldwide, best practice recommendations for achieving this goal include a sedation protocol based on the use of clinical sedation scales $^{4}$ or daily interruption of sedatives, ${ }^{3}$ or both. However, such sedation practices are applied in less than a third of ICUs throughout the world, ${ }^{9-12}$ making them one of the least applied ICU practice recommendations. ${ }^{13}$ Thus, according to guidelines, most mechanically ventilated patients are still oversedated..$^{14,15} \mathrm{~A}_{\text {study }}{ }^{16}$ in 1884 mechanically ventilated ICU patients showed that patients admitted to the ICU after unplanned surgery were twice as often oversedated during the first $48 \mathrm{~h}$ compared with patients admitted after elective surgery.

Severe pain can lead to specific issues in the management of sedation and analgesia in postoperative ICU patients. Strøm and colleagues ${ }^{17}$ showed that a trial of no sedation within $24 \mathrm{~h}$ after intubation in a mixed medical and surgical population of ICU patients was associated with an increase in days spent without
Lancet Respir Med 2017 Published Online September 18, 2017 http://dx.doi.org/10.1016/ S2213-2600(17)30304-1 See Online/Comment http://dx.doi.org/10.1016/ S2213-2600(17)30350-8

*SOS-Ventilation study investigators are listed in the appendix

Department of Anaesthesia and Intensive Care, Montpellier University Saint Eloi Hospital, Montpellier, France (Prof G Chanques MD $M$ Conseil MD, A Prades RN $J$ Carr MD, B Jung MD,

$F$ Belafia MD, $M$ Cissé MD J-M Delay MD, A de Jong MD, Prof S Jaber MD); Department of Medical Information, Hôpital de la Colombière, Montpellier, France $(G$ Mercier MD N Molinari PhD); PhyMedExp, University of Montpellier INSERM U1046, CNRS UMR 9214, Montpellier, France (Prof G Chanques, B Jung, A de Jong, Prof S Jaber, N Molinari); Department of Anaesthesia, Intensive Care, Pain and Emergency, Hôpital Carémeau, CHU de Nîmes, Nîmes, France (C Roger MD, L Muller MD, ProfJ-Y Lefrant MD); GReD, UMR/CNRS6293, INSERM U1103, Université Clermont Auvergne, Clermont-Ferrand France (Prof)-M Constantin MD, Prof E Futier MD); and Department of Perioperative Medicine, Hôpital Estaing, $\mathrm{CHU}$ de Clermont-Ferrand, ClermontFerrand, France (Prof)-M Constantin, Prof E Futier) Correspondence to: Prof Gérald Chanques, Department of Anaesthesia and Intensive Care, Montpellie University Saint Eloi Hospital, Montpellier F-34295, CEDEX 5, France g-chanques@chu-montpellier.fr See Online for appendix 
Research in context

\section{Evidence before this study}

We searched MEDLINE and ClinicalTrials.gov from their inception to March 21, 2017. The search term equation was: ((ICU) or (critical care)) and ((sedation) or (sedatives) or (analgesia) or (analgesics) or (pain) or (agitation) or (delirium) or (mechanical ventilation)). Studies were included if they evaluated an intervention concerning sedation practices aimed at decreasing sedation in intensity (ie, level of sedation) or duration, or both, in adult patients aged 18 years or older and admitted to an intensive care unit (ICU). We found one randomised controlled trial assessing the effect of early interruption of sedation in a mixed medical and surgical population of ICU patients within $24 \mathrm{~h}$ after intubation. The no-sedation group had a significant increase in days without ventilation and a decrease in days in the ICU and hospital compared with the control group. After completion of our trial, a meta-analysis of six randomised controlled trials of protocolised sedation in medical or mixed medical and surgical ICU patients reported a significant association between protocolised sedation and a reduction in overall mortality and length of stay in ICU and hospital compared with usual care. The quality of the evidence was moderate for all six trials. However, none of these trials exclusively enrolled postoperative surgical ICU patients; also, enrolment occurred after a 24-48 h period of mechanical ventilation. Thus, we identified a need for an interventional study on sedation practices during the early period of mechanical ventilation.

\section{Added value of this study}

In this study, sedation was decreased in postoperative, critically ill ICU patients much sooner than in previous studies. Sedation was interrupted as soon as possible after admission to the ICU from the operating theatre. Patients in the intervention group had a median time of less than $2 \mathrm{~h}$ of sedation. Unnecessary deep and prolonged sedation in the control group (where median sedation time was $33 \mathrm{~h}$ ) was also avoided to prevent iatrogeny. The effect of this immediate interruption of sedation was a significantly shorter median time to successful extubation in the intervention group than in the control group ( $8 \mathrm{~h}$ vs $50 \mathrm{~h}$ ). Moreover, this study is, to our knowledge, the first randomised controlled trial evaluating a sedation intervention that showed a positive effect on delirium. The findings of our study reinforce the rationale for interrupting sedation as early as possible in ICU patients.

Implications of all the available evidence

Given the clinical and economic burden of critical illness, postoperative morbidity, and the substantial number of patients who could benefit from this strategy, sedation should be immediately interrupted in postoperative patients admitted to an ICU. Further studies should be done to investigate whether such an immediate cessation of sedation would have similar effects in medical ICU and postoperative ICU patients with a more severe acute respiratory failure at time of admission or patients undergoing surgery other than abdominal surgery. ventilation and a decrease in days spent in the ICU and hospital, but also with an increased incidence of delirium and antipsychotic requirements. The effect of early interruption of sedatives in postoperative patients remains unknown. Although pain might necessitate high analgesia and sedation requirements, anaesthesia could worsen overall clinical status.

We hypothesise that certain critically ill patients admitted to an ICU following surgery might not actually require sedation and ventilation at all, and that avoidance of continuous sedation as soon as possible would be a feasible strategy associated with improved outcomes. Because of the substantial number of postoperative patients sedated for mechanical ventilation in the ICU in whom avoidance of continuous sedation could be a feasible intervention, we did the Strategy of Optimized Sedation-Ventilation (SOS-Ventilation) study to evaluate whether immediate cessation of sedation could improve postoperative outcomes compared with usual sedation care.

\section{Methods \\ Study design}

The SOS-Ventilation study was an investigator-initiated, multicentre, stratified, parallel-group clinical trial with a computer-generated allocation sequence and centralised randomisation. In accordance with French law, the study protocol and statistical analysis plan were approved for all centres by the local ethics committee (Comité de Protection des Personnes Sud-Méditerranée IV, Montpellier, France). The full protocol is provided in the appendix. The trial was done in accordance with the Declaration of Helsinki and was registered on Nov 10, 2011 (ClinicalTrials.gov, number NCT01486121). Three ICU centres participated in the study, with a patient to nurse ratio of 2.5 to 1 and a patient to assistant-nurse ratio of 4 to 1 (patient to bedside caregiver ratio of 1.5 to 1). Unlike the randomised trial by Strøm and colleagues, ${ }^{17}$ no extra individuals (such as an assistant nurse or a family member) were required to reassure the non-sedated patients specifically for the purpose of the present trial.

\section{Patients}

Patients with national health-care insurance, who were older than 18 years, intubated and mechanically ventilated in volume assist-control mode in ICUs less than $24 \mathrm{~h}$ after a surgery, for an expected $12 \mathrm{~h}$ mechanical ventilation, with at least one organ dysfunction defined by a Sequential Organ Failure Assessment (SOFA) score $^{18}$ greater than 1 for any organ, were eligible for participation as soon as they were postoperatively normothermic (body temperature $>36^{\circ} \mathrm{C}$ ) without any residual paralysis induced by neuromuscular blocking 
agents potentially used for anaesthesia, as clinically assessed by the head lift test or with a monitoring device according to local practices.

Patients who had been admitted to the hospital ICU for more than 7 days before surgery, had brain injury, had severe acute respiratory distress syndrome (ARDS, as previously defined in the ACURASYS trial ${ }^{19}$ by a ratio of the partial pressure of arterial oxygen to the fraction of inspired oxygen of less than 150), had a history of drug abuse, had withdrawal of care, were under guardianship, were pregnant, were enrolled in another trial evaluating sedation or ventilation, or had a surgical contraindication to discontinuing sedation (ie, uncontrolled bleeding, surgical re-intervention planned within $24 \mathrm{~h}$, or open abdominal wall) were excluded. The trial was interrupted if patients or their proxies declined participation or if brain injury occurred after enrolment.

Taking into account the fact that patients would be sedated following ICU admission, and that certain admissions would occur after an unplanned surgery under emergency conditions, complete adherence to patient consent procedures was deemed impossible before surgery or following ICU admission. In accordance with French law and with the approval of the ethics committee in favour of the research objective, a consent dispensation for emergency situations was enabled to minimise the time between ICU admission and randomisation as much as possible. Written consent to continue the research and analyse the data was obtained from the patient or their proxies as soon as possible.

\section{Randomisation}

Patients were screened and underwent randomisation between Dec 2, 2011, and Feb 27, 2014. Patients were randomly assigned (1:1 ratio) by stratified randomisation with minimisation via a restricted web platform. Randomisation was stratified according to centre, the patient's age ( $<60$ years or $\geq 60$ years), and illness severity assessed by the Simplified Acute Physiological Score (SAPS) II $^{20}$ (SAPS II score $<38$ or $\geq 38$ ). These cutoffs were determined on the basis of the mean scores observed in our ICU population. ${ }^{21}$ The SAPS II score used for stratification randomisation took into account the worst value available up to the previous $24 \mathrm{~h}$ from randomisation.

\section{Procedures}

Patients were assigned to receive either continuous sedation for tolerance of assist-control ventilation according to recommended guidelines (control group) or an immediate interruption of sedation (intervention group). In the intervention group, when anxiety, agitation, pain, discomfort, polypnoea, or patient-ventilator asynchrony persisted after management optimisation according to established protocols (appendix pp 5-9), continuous sedation was used for $6 \mathrm{~h}$. If more than two periods of sedation were required within $24 \mathrm{~h}$, continuous sedation was prolonged until the next day.
In both groups, sedation was standardised according to recommended guidelines ${ }^{7,22}$ to minimise the risk of oversedation. A previously published sedation analgesia protocol $^{11}$ was used by bedside nurses every $4 \mathrm{~h}$ and sedation levels and pain intensity were assessed with the Richmond Agitation Sedation Scale (RASS) ${ }^{23,24}$ and the Behavioral Pain Scale (BPS). ${ }^{25}$ Sedation was primarily targeted at a light or moderate level (RASS between -1 and -3 ). Sedatives were interrupted daily every morning according to criteria selected by French intensive

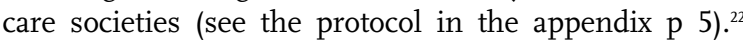
After interruption of sedation, agitation was again assessed every 4-8 $\mathrm{h}$ until discharge from the ICU by the RASS in all patients (sedated and non-sedated, intubated and non-intubated). Pain was assessed either by the BPS in patients unable to communicate or by the visually enlarged 0-10 Numeric Rating Scale (NRS) ${ }^{26}$ in those able to communicate. Diagnosis and therapeutic management of pain and agitation were standardised according to published protocols. ${ }^{27,28}$ Major opioid infusions (ie, sufentanil or remifentanil) were stopped at the same time as sedatives. Postoperative analgesia was provided by use of a multimodal strategy with paracetamol, nefopam, and tramadol (appendix p 9). Major opioids were reintroduced if multimodal analgesia was insufficient for treatment of basal pain, or for prevention and treatment of procedural pain. ${ }^{27,28}$ When severe ARDS occurred after randomisation, deep sedation was reintroduced at the same time as a neuromuscular blocking agent (cisatracurium) for a maximum of $48 \mathrm{~h}$, according to the ACURASYS sedation protocol. ${ }^{19}$ Patients who developed severe ARDS were not excluded from the trial or the analysis.

Ventilators were set according to a lung protective ventilation strategy in both groups. ${ }^{29}$ Pressure support ventilation, a mode allowing for spontaneous breathing and less asynchrony, ${ }^{30}$ was used after interruption of sedation. Sedation interruption was coupled with a spontaneous breathing trial ${ }^{31}$ on at least a daily basis. Extubation was implemented in accordance with standardised criteria, as recommended ${ }^{32}$ (appendix p 5).

\section{Outcomes}

The primary outcome measure was time to successful extubation, defined as the time from randomisation to the time of extubation (or tracheotomy mask) for at least $48 \mathrm{~h}$, as previously defined ${ }^{8}$ (the time of extubation corresponds to the beginning of a $48 \mathrm{~h}$ tube-free period). This outcome was centrally assessed. Secondary outcomes calculated on day 28 were the number of ventilator-free days (ie, days alive and without invasive mechanical ventilation between day 1 and day 28), the duration of ICU and hospital stays, and mortality. Secondary outcome reporting included the following health-care-related complications during the ICU stay: coma as defined by a RASS of -4 or -5 ; delirium as defined by the Confusion Assessment Method for the Intensive Care Unit (CAM-ICU) $;{ }^{33}$ pain as defined by a BPS score of more than 3 (or a self-reported 0-10 NRS $>3$ in 


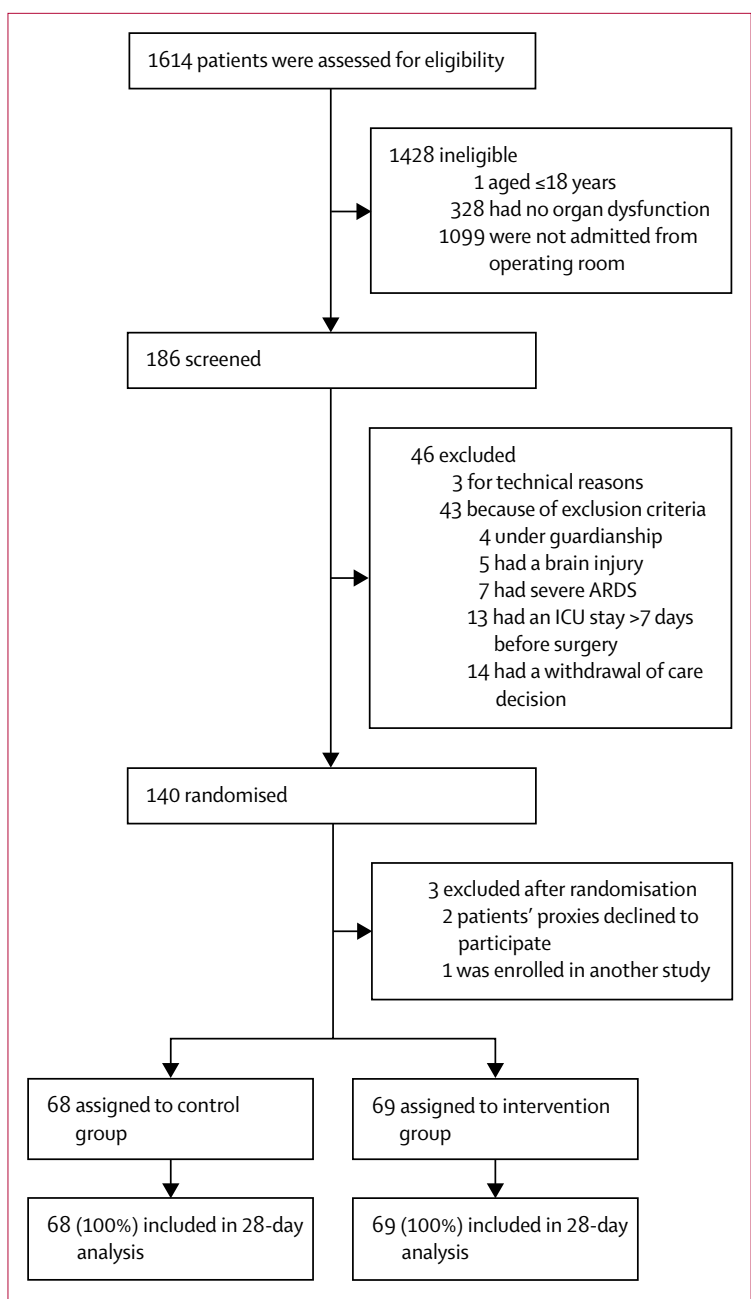

Figure 1: Trial profile

Technical reasons preventing enrolment were related to a high workload in the intensive care unit (ICU). ARDS=acute respiratory distress syndrome.

patients able to communicate), evaluated by the research team every morning; ileus; pressure ulcer; self-removal of medical devices; surgical re-intervention; health-careassociated infections (for definitions, see the appendix $\mathrm{p}$ 10), and health-care resource utilisation (type, duration, and dose of sedatives and analgesics, and vasopressor (norepinephrine) use within the previous $24 \mathrm{~h}$, with highdose norepinephrine defined as $>0.1 \mu \mathrm{g} / \mathrm{kg}$ per min according to the SOFA score). The characteristics of mechanical ventilation, including the use of non-invasive ventilation, were assessed by the research team every morning for the previous $24 \mathrm{~h}$. Long-term outcomes including mortality, post-traumatic stress disorder, anxiety, depression, and quality of life at 3 months and at 12 months were also assessed as prespecified secondary outcomes.?

\section{Statistical analysis}

We calculated that a sample size of 140 patients would provide a power of $80 \%$ to detect an absolute between-group difference of $72 \mathrm{~h}$ with an SD of $140 \mathrm{~h}$ in the primary outcome at a two-sided alpha level of $0 \cdot 05$.

All analyses were done on data from the modified intention-to-treat population, which included all patients who underwent randomisation except for those who were excluded after randomisation. Group medians for the primary outcome were compared by use of the Mann-Whitney $U$ test (the day of extubation was considered as the day of death for patients who died while still intubated). Hazard ratios (HRs) with 95\% CIs were calculated by use of multivariate Cox regression to take into account the censored primary outcome for deceased patients. Stepwise selection was used to determine the final Cox regression model $(\mathrm{p}<0 \cdot 10$ to enter the model, $\mathrm{p}<0 \cdot 05$ to remain in the final model, in addition to randomisation stratification parameters). A competing risk model for the competing risk of death was used as a sensitivity analysis. A secondary analysis of the primary outcome measure involving a bootstrapped $t$ test (frailtypack in $\mathrm{R}$ ) was also done to support the findings of our original analysis.

For secondary outcomes, continuous variables were compared with the unpaired $t$ test or the Mann-Whitney $\mathrm{U}$ test; categorical variables were compared with the $\chi^{2}$ test or Fisher's exact test, as appropriate (univariate analysis).

For the time-to-extubation analysis, the event occurred when a patient was extubated within 28 days from randomisation and remained extubated for more than $48 \mathrm{~h}$. Patients who died before extubation were censored at death. The time-to-event curves were calculated for hospital discharge by use of the same method. All analyses were done with $\mathrm{R}$ statistical software, version 3.0.1, and SAS, version 9.3. A two-sided $p$ value of less than 0.05 was considered to indicate statistical significance.

This study is registered with ClinicalTrials.gov, number NCT01486121.

\section{Role of the funding source}

The funder of the study had no role in study design, data collection, data analysis, data interpretation, or writing of the report. The corresponding author had full access to all the data in the study and had final responsibility for the decision to submit for publication.

\section{Results}

From Dec 2, 2011, to Feb 27, 2014, 1614 ICU patients were assessed for eligibility. Among the 186 eligible patients, 137 were included in the intention-to-treat analysis and were followed up for 28 days (figure 1). Data for the primary outcome measure were available for all patients. Of the 137 patients, 68 were randomly assigned to the control group and 69 to the intervention group. Following inclusion, baseline characteristics were similar in both groups (table 1; appendix pp 11-15). All patients were admitted to ICU after abdominal surgery, primarily for septic shock. 


\begin{tabular}{|c|c|c|}
\hline & $\begin{array}{l}\text { Control group } \\
(n=68)\end{array}$ & $\begin{array}{l}\text { Intervention } \\
\text { group }(n=69)\end{array}$ \\
\hline Age, years & $67(57-78)$ & $70(59-76)$ \\
\hline Men & $40(59 \%)$ & $41(59 \%)$ \\
\hline Women & $28(41 \%)$ & $28(41 \%)$ \\
\hline Body-mass index, $\mathrm{kg} / \mathrm{m}^{2}$ & $26(22-29)$ & $26(23-29)$ \\
\hline \multicolumn{3}{|l|}{ Comorbidities } \\
\hline Current smoker & $13(19 \%)$ & $12(17 \%)$ \\
\hline Diabetes & $13(19 \%)$ & $12(17 \%)$ \\
\hline Alcohol intake & $8(12 \%)$ & $9(13 \%)$ \\
\hline Hypertension & $32(47 \%)$ & $35(51 \%)$ \\
\hline Coronary disease & $12(18 \%)$ & $12(17 \%)$ \\
\hline Chronic heart failure & $11(16 \%)$ & $8(12 \%)$ \\
\hline Chronic kidney disease & $8(12 \%)$ & $3(4 \%)$ \\
\hline Cirrhosis & $1(1 \%)$ & $3(4 \%)$ \\
\hline $\begin{array}{l}\text { Chronic obstructive pulmonary } \\
\text { disease }\end{array}$ & $9(13 \%)$ & $11(16 \%)$ \\
\hline Immunosuppression & $6(9 \%)$ & $4(6 \%)$ \\
\hline Haematological malignancy & $1(1 \%)$ & $2(3 \%)$ \\
\hline Cancer & $26(38 \%)$ & $25(36 \%)$ \\
\hline Psychoactive treatment & $12(18 \%)$ & $11(16 \%)$ \\
\hline Opioid treatment & $4(6 \%)$ & $3(4 \%)$ \\
\hline Serum creatinine, $\mu \mathrm{mol} / \mathrm{L}^{*}$ & $102(75-178)$ & $117(79-151)$ \\
\hline Abdominal surgery & $68(100 \%)$ & $69(100 \%)$ \\
\hline Unplanned surgery & $61(90 \%)$ & $64(93 \%)$ \\
\hline \multicolumn{3}{|l|}{ Indication for unplanned surgery } \\
\hline Intra-abdominal infection & $43 / 61(70 \%)$ & $43 / 64(67 \%)$ \\
\hline Occlusion & $4 / 61(7 \%)$ & $8 / 64(13 \%)$ \\
\hline Ischaemia & $5 / 61(8 \%)$ & $6 / 64(9 \%)$ \\
\hline Haemorrhage & $5 / 61(8 \%)$ & $6 / 64(9 \%)$ \\
\hline Other & $4 / 61(7 \%)$ & $1 / 64(2 \%)$ \\
\hline Planned surgery & $7 / 68(10 \%)$ & $5 / 69(7 \%)$ \\
\hline \multicolumn{3}{|l|}{ Indication for planned surgery } \\
\hline Cancer & $5 / 7(71 \%)$ & $5 / 5(100 \%)$ \\
\hline Other & $2 / 7(29 \%)$ & $0 / 5(0 \%)$ \\
\hline \multicolumn{3}{|l|}{ Type of surgery } \\
\hline Peritoneal cleansing and drainage & $25(37 \%)$ & $17(25 \%)$ \\
\hline Colorectal resection & $15(22 \%)$ & $17(25 \%)$ \\
\hline Small intestine resection & $8(12 \%)$ & $7(10 \%)$ \\
\hline Cholecystectomy & $5(7 \%)$ & $8(11 \%)$ \\
\hline Duodenopancreatectomy & $2(3 \%)$ & $4(6 \%)$ \\
\hline \multirow[t]{2}{*}{ Other } & $13(19 \%)$ & $16(23 \%)$ \\
\hline & \multicolumn{2}{|c|}{ (Table 1 continues in next column) } \\
\hline
\end{tabular}

Median time between randomisation and successful extubation (the primary outcome) was significantly lower in the intervention group than in the control group (table 2; figure 2). A multivariate analysis of variables associated with the primary outcome included variables for which a $\mathrm{p}$ value was less than $0 \cdot 10$ after univariate analysis (SOFA score, history of hypertension, opioid treatment, Knauss chronic health status, reason for unplanned surgery, procedural severity scores, and variables at ICU admission: serum lactate, vasopressors, midazolam, propofol, propofol dose, RASS, BPS, and

\begin{tabular}{|c|c|c|}
\hline & $\begin{array}{l}\text { Control group } \\
(n=68)\end{array}$ & $\begin{array}{l}\text { Intervention } \\
\text { group }(n=69)\end{array}$ \\
\hline \multicolumn{3}{|l|}{ (Continued from previous column) } \\
\hline Work Relative Value Units (RVUs) & $27(20-28)$ & $25(18-30)$ \\
\hline Procedural Severity Score†, morbidity & $91(85-93)$ & $90(83-93)$ \\
\hline Procedural Severity Score $\dagger$, mortality & $78(67-78)$ & $73(59-78)$ \\
\hline Duration of surgery, $\mathrm{h}$ & $2(1-4)$ & $2(1-4)$ \\
\hline $\begin{array}{l}\text { Intraoperative fluid administration, } \\
\mathrm{mL} / \mathrm{kg} \text { per } \mathrm{h} \neq\end{array}$ & $14(9-25)$ & $17(10-24)$ \\
\hline $\begin{array}{l}\text { Time between admission from } \\
\text { operating room and randomisation, } \mathrm{h}\end{array}$ & $4(1-6)$ & $2(1-6)$ \\
\hline \multicolumn{3}{|l|}{ Clinical status at entry into the study } \\
\hline SAPSIIS & $45(38-53)$ & $47(37-57)$ \\
\hline SOFA total score & $8(6-9)$ & $8(6-10)$ \\
\hline Cardiovascular subscore & $4(4-4)$ & $4(4-4)$ \\
\hline Respiratory subscore & $2(1-3)$ & $2(2-3)$ \\
\hline Renal subscore & $1(0-2)$ & $1(0-2)$ \\
\hline Liver subscore & $0(0-2)$ & $0(0-2)$ \\
\hline Coagulation subscore & $0(0-1)$ & $0(0-1)$ \\
\hline Neurological subscore & $0(0-0)$ & $0(0-0)$ \\
\hline Use of vasopressors (norepinephrine) & $53(78 \%)$ & $58(84 \%)$ \\
\hline Serum lactate, $\mathrm{mmol} / \mathrm{L}$ & $3(2-4)$ & $3(2-6)$ \\
\hline \multicolumn{3}{|c|}{$\begin{array}{l}\text { Data are } n(\%) \text { or median (IQR), unless otherwise stated. The sum of percentages } \\
\text { is different from } 100 \% \text { because there could be several reasons for ICU admission. } \\
\text { ICU=intensive care unit. *Serum creatinine was available before surgery in } \\
51 \text { patients in the control group and in } 54 \text { in the intervention group. †Procedural } \\
\text { Severity Scores were calculated according to Dalton and colleagues' method. }{ }^{34} \\
\text { łIntraoperative fluid administration was available in } 55 \text { patients in each group, } \\
\text { calculated according to Shin and colleagues' method. }{ }^{35} \text { SThe Simplified Acute } \\
\text { Physiology Score (SAPS) II is based on } 17 \text { variables; scores range from } 0 \text { to } 163 \text {, } \\
\text { with increasing scores indicating increased disease severity. }{ }^{20} \text { TThe Sequential } \\
\text { Organ Failure Assessment (SOFA) score includes subscores ranging from } 0 \text { to } 4 \text { for } \\
\text { each of six components (circulation, lungs, kidneys, liver, coagulation, and CNS). } \\
\text { The SOFA neurological score was calculated on the basis of a clinical assessment } \\
\text { before anaesthesia induction. Aggregated scores range from } 0 \text { to } 20 \text {, with higher } \\
\text { scores indicating more severe organ failure. }{ }^{18}\end{array}$} \\
\hline
\end{tabular}

plateau airway pressure) and stratification variables (centre, age, SAPS II). Results of the univariate and multivariate analysis are shown in the appendix (pp 16-19). There were three independent variables remaining in the final model: intervention, SAPS II, and plateau airway pressure at admission. The intervention was significantly associated with reduced time to successful extubation (adjusted HR 5 2; 95\% CI 3 1-8 8 , $\mathrm{p}<0.0001$; table 2). The adjusted HR for the competing risk of death was $3 \cdot 5$ (95\% CI 2.2-5.6, p<0.0001; sensitivity analysis), and the bootstrapped modelling of the adjusted HR was $6 \cdot 0(3 \cdot 3-13 \cdot 0 ; \mathrm{p}<0 \cdot 0001$; table 2$)$.

The log-rank test for between-group differences applied to the Kaplan-Meier estimates for the primary outcome yielded a $\mathrm{p}$ value of less than 0.0001 in favour of the intervention group (appendix p 27).

Fewer patients in the intervention group had a coma or delirium compared with the control group (table 2). There were significantly more delirium-free days in the intervention group than in the control group (median 
26 days [IQR 24-27] vs median 28 days [26-28], $\mathrm{p}=0 \cdot 002)$.

There were no significant differences between groups for the other complications observed in the ICU (pain, self-removal of medical devices, ileus, pressure ulcers, health-care-associated infections, and surgical re-interventions).

\begin{tabular}{|c|c|c|c|c|}
\hline & $\begin{array}{l}\text { Control } \\
\text { group } \\
(n=68)\end{array}$ & $\begin{array}{l}\text { Intervention } \\
\text { group } \\
(n=69)\end{array}$ & $\begin{array}{l}\text { Group difference }{ }^{*} \\
(95 \% \mathrm{Cl})\end{array}$ & p value \\
\hline \multicolumn{5}{|l|}{ Primary outcome } \\
\hline $\begin{array}{l}\text { Time between randomisation and } \\
\text { successful extubation, } \mathrm{h}\end{array}$ & $50(29-93)$ & $8(4-36)$ & $-33 \cdot 6(-44 \cdot 9$ to $-22 \cdot 4)$ & $<0.0001$ \\
\hline Adjusted hazard ratio $(95 \% \mathrm{Cl})$ & .. &.. & $5 \cdot 2(3 \cdot 1-8 \cdot 8)$ & $<0.0001$ \\
\hline $\begin{array}{l}\text { Adjusted hazard ratio for competing } \\
\text { risk of death }(95 \% \mathrm{Cl})\end{array}$ &.. &.. & $3 \cdot 5(2 \cdot 2-5 \cdot 6)$ & $<0.0001$ \\
\hline Bootstrap $t$ test $(95 \% \mathrm{Cl})$ & .. &.. & $6 \cdot 0(3 \cdot 3-13 \cdot 0)$ & $<0.0001$ \\
\hline \multicolumn{5}{|c|}{ Secondary outcome measures at day 28} \\
\hline \multicolumn{5}{|c|}{ Neurological complications and pain in the ICU } \\
\hline Coma† & $34(50 \%)$ & $15(22 \%)$ & $-28(-45$ to -11$)$ & 0.0006 \\
\hline Days with coma & $1(0-2)$ & $0(0-0)$ & $-0.5(-1 \cdot 0$ to 0.0$)$ & 0.0008 \\
\hline Delirium $\neq$ & $48(72 \%)$ & $28(43 \%)$ & $-29(-50$ to -14$)$ & 0.0004 \\
\hline Days with delirium & $2(0-4)$ & $0(0-2)$ & $-0.5(-1 \cdot 0$ to 0.0$)$ & 0.003 \\
\hline Severe pain $\S$ & $14(21 \%)$ & $14(20 \%)$ & $-0.6(-16$ to 14$)$ & 0.93 \\
\hline Days with severe pain & $0(0-0)$ & $0(0-0)$ & $0.0(-1.0$ to 1.0$)$ & 0.95 \\
\hline Moderate pain $\S$ & $41(61 \%)$ & $38(55 \%)$ & $-6(-24$ to 12$)$ & 0.47 \\
\hline Days with moderate pain & $1(0-2)$ & $1(0-1)$ & $0.0(0.0$ to 0.0$)$ & 0.62 \\
\hline \multicolumn{5}{|l|}{ Other complications in ICU } \\
\hline lleus & $2(3 \%)$ & $2(3 \%)$ & -0 (-7 to 7$)$ & 1.00 \\
\hline Pressure ulcer $>5 \mathrm{~cm}$ & $0(0 \%)$ & $0(0 \%)$ & NA & NA \\
\hline Self-extubation & $0(0 \%)$ & $1(1 \%)$ & NA & 1.00 \\
\hline Self-removal of catheter & $2(3 \%)$ & $2(3 \%)$ & $-0(-7$ to 7$)$ & 1.00 \\
\hline Surgical re-intervention & $12(18 \%)$ & $12(17 \%)$ & -0 (-15 to 14$)$ & 0.91 \\
\hline \multicolumn{5}{|c|}{ Health-care-associated infections in ICU and hospital } \\
\hline $\begin{array}{l}\text { At least one health-care-associated } \\
\text { infection }\end{array}$ & $9(13 \%)$ & $7(10 \%)$ & $-3(-10$ to 4$)$ & 0.57 \\
\hline Pneumonia & $2(3 \%)$ & $1(1 \%)$ & NA & NA \\
\hline Bacteraemia & $6(9 \%)$ & $5(7 \%)$ & NA & NA \\
\hline Urinary tract infection & $1(1 \%)$ & $1(1 \%)$ & NA & NA \\
\hline $\begin{array}{l}\text { Central venous catheter } \\
\text { colonisation }\end{array}$ & $1(1 \%)$ & $0(0 \%)$ & NA & NA \\
\hline \multicolumn{5}{|l|}{ Resource utilisation } \\
\hline $\begin{array}{l}\text { Total duration of mechanical } \\
\text { ventilation, } \mathrm{h}\end{array}$ & $55(29-103)$ & $9(4-40)$ & $-34 \cdot 4(-46 \cdot 5$ to $-22 \cdot 3)$ & $<0.0001$ \\
\hline Ventilator-free days & $25(13-27)$ & $27(10-28)$ & $1.4(0.6$ to $2 \cdot 1)$ & 0.0004 \\
\hline $\begin{array}{l}\text { Ventilator-free days with deceased } \\
\text { accounting for } 0\end{array}$ & $25(0-27)$ & $27(4-28)$ & $1.1(0.4$ to 1.8$)$ & 0.0006 \\
\hline Extubation & $58(85 \%)$ & $63(91 \%)$ & $6(-6$ to 18$)$ & 0.27 \\
\hline Re-intubation & $12 / 58(21 \%)$ & $18 / 63(29 \%)$ & 8 (-8 to 19$)$ & 0.32 \\
\hline Tracheotomy & $1(1 \%)$ & $3(4 \%)$ & $3(-4$ to 10$)$ & 0.62 \\
\hline Non-invasive ventilation & $36(53 \%)$ & $33(48 \%)$ & $-5(-23$ to 13$)$ & 0.55 \\
\hline Days of non-invasive ventilation & $4(2-6)$ & $4(2-5)$ & $-0.5(-2.0$ to 1.0$)$ & 0.58 \\
\hline Use of norepinephrine & $50(74 \%)$ & $50(73 \%)$ & $-1(-18$ to 14$)$ & $0 \cdot 77$ \\
\hline $\begin{array}{l}\text { Days with norepinephrine dose } \\
>0.1 \mu \mathrm{g} / \mathrm{kg} \text { per min }\end{array}$ & $2(2-4)$ & $1(0-3)$ & $-0.5(-1 \cdot 0$ to $0 \cdot 0)$ & 0.04 \\
\hline
\end{tabular}

(Table 2 continues on next page)
The use of prophylactic or curative postoperative noninvasive ventilation did not differ significantly between groups (33 [48\%] of 69 in the intervention group vs 36 [53\%] of 68 in the control group; absolute difference $-5 \%, \mathrm{p}=0 \cdot 55)$.

Other health-care resource utilisation was significantly reduced in the intervention group, with an increased number of ventilator-free days, fewer days on high-dose vasopressors (norepinephrine), and a higher probability of being discharged at day 28 (table 2; figure 3). Day 28 mortality did not differ significantly between the two groups (table 2). No significant difference between the two groups was observed for mortality, post-traumatic stress disorder, anxiety, depression, and quality of life at 3 months and 12 months (appendix pp 25, 26).

After randomisation, continuous sedation was stopped according to the protocol after a median time of $15 \mathrm{~min}$ in the intervention group and $33 \mathrm{~h}$ in the control group (table 3). Sedation was resumed in less than $30 \%$ of patients in both groups, mainly due to patient-ventilator asynchrony (62\%) or surgical re-intervention (26\%), without any significant difference between groups. Seven patients in the control group had a protocol deviation with an immediate interruption of sedation, and one patient in the intervention group had a longer duration of sedation than expected. The appendix (pp 20, 21) describes the drugs used for sedationanalgesia after randomisation in both groups. As expected, according to the research protocol, sedatives and major opioids were used significantly more often in the control group than in the intervention group, whereas minor opioids (eg, tramadol) were used significantly more often in the intervention group. There were no significant differences between groups in the use of other drugs (eg, neuromuscular-blocking agents, antipsychotics, and anxiolytics).

\section{Discussion}

In this multicentre, randomised controlled trial of critically ill postoperative ICU patients after abdominal surgery, immediate interruption of sedation and early use of spontaneous ventilation decreased the time to extubation compared with usual sedation care. Immediate interruption of sedation also led to significant decreases in coma, delirium, and high-dose vasopressor use. Patients who received the intervention had a significantly higher number of ventilator-free days and a higher probability of being discharged at day 28 than did patients who received usual care.

Times to extubation reported in both groups in our study are much lower than those reported in previous randomised controlled trials evaluating sedation practices..$^{8,1736}$ There are two main reasons that might help explain these differences: the duration of sedation before enrolment was over $48 \mathrm{~h}$ in most previous studies, whereas in our study enrolment occurred immediately after ICU admission (following a surgical 
procedure); and the population in this study differed from those of previous studies (mainly medical in the other studies and exclusively surgical in our study). However, illness severity as evaluated by the SAPS II and SOFA scores was similar between our study and previous studies. Additionally, time to extubation is dependent on sedation practices, as shown in this study, and sedation practices can differ between surgical and medical ICU patients. However, there is a paucity of data about sedation in postoperative ICU patients. The study by Schaller and colleagues ${ }^{37}$ evaluating an early mobilisation programme for postoperative ICU patients reported a duration of sedation of 4-5 days within 28 days. In our study, both groups had much lower durations of sedation (1-2 days). This decrease is explained by careful decision making in sedation management, including daily interruptions in accordance with the study objectives and design.

Most patients in our study were admitted directly to the ICU for septic shock after abdominal surgery. Intra-abdominal infection is the second leading cause of ICU admission (after complicated pneumonia), accounting for almost $10 \%$ of all ICU patients, ${ }^{38,39}$ and almost $20 \%$ of all patients with infections. ${ }^{39}$ It is the most common cause of infection $(70 \%)$ in septic patients admitted to a surgical ICU. ${ }^{40}$ In patients with septic shock, sedation is often required to manage invasive treatments and to enable tolerance to mechanical ventilation, improve gas exchange, control agitation and pain, and therefore improve tissue oxygenation. ${ }^{41}$ A fundamental reason for our trial was to ascertain whether the sustained use of sedatives during recovery after surgery might be unnecessary and lead to increased iatrogenic morbidity and increased resource utilisation. Our hypothesis was that in this specific population of critically ill patients in the postoperative period of abdominal surgery, haemodynamic instability could be, in part, the consequence of the vasodilator side-effects of anaesthesia drugs. After transfer to the ICU, anaesthesia is continued to some extent by intravenous sedatives (continuous sedation), until the patient recovers from hypothermia and neuromuscular blockade. After this point, the benefits of sedation are unclear. The vasodilator effects of sedation, especially propofol, might interfere with the course of disease itself (sepsis), thus artificially worsening the clinical condition. ${ }^{42-44}$ In support of this hypothesis, we observed a significant reduction in the duration of high-dose vasopressor use in the intervention group, although illness severity (as assessed by SAPS II and SOFA scores) and serum lactate did not differ significantly between the groups at enrolment (appendix $\mathrm{p}$ 13). This study showed that, to test related effects on the overall clinical picture for a given patient, sedation should be stopped as soon as possible, immediately after the patient's transfer from the operating room. We showed that resumption of sedation was unnecessary in more than $70 \%$ of these patients. The 2012 International

\begin{tabular}{|c|c|c|c|c|}
\hline & $\begin{array}{l}\text { Control } \\
\text { group } \\
(n=68)\end{array}$ & $\begin{array}{l}\text { Intervention } \\
\text { group } \\
(n=69)\end{array}$ & $\begin{array}{l}\text { Group difference } \\
(95 \% \mathrm{Cl})\end{array}$ & p value \\
\hline \multicolumn{5}{|l|}{ (Continued from previous page) } \\
\hline Days in ICU & $6(3-11)$ & $5(3-9)$ & $-0.9(-2.5$ to 0.6$)$ & 0.879 \\
\hline Days in ICU (survivors only) & $7(4-10)$ & $4(3-8)$ & $-1 \cdot 2(-2 \cdot 9$ to 0.5$)$ & $0.78 \Phi$ \\
\hline Days in hospital & $25(11-28)$ & $17(10-27)$ & $-2 \cdot 2(-4 \cdot 5$ to $0 \cdot 1)$ & $0.04 \mathbb{\top}$ \\
\hline Days in hospital (survivors only) & $27(17-28)$ & $23(14-27)$ & $-2 \cdot 0(-3 \cdot 9$ to $-0 \cdot 1)$ & 0.019 \\
\hline \multicolumn{5}{|l|}{ Mortality } \\
\hline ICU mortality & $14(21 \%)$ & $13(19 \%)$ & $-2(-17$ to 13$)$ & 0.97 \\
\hline Overall mortality & $17(25 \%)$ & $17(25 \%)$ & $-0(-16$ to 16$)$ & 0.99 \\
\hline \multicolumn{5}{|c|}{$\begin{array}{l}\text { Data are } n(\%) \text { or median (IQR), unless otherwise stated. ICU=intensive care unit. NA=not applicable. *Group difference } \\
\text { refers to the intervention group value minus control group value: absolute difference (\%) or Hodges-Lehmann median } \\
\text { difference. †Coma was defined by a Richmond Agitation Sedation Scale (RASS) of }-4 \text { or }-5 \text {, assessed by the research } \\
\text { team every morning. } \neq \text { Delirium was defined by a positive score on the Confusion Assessment Method for the Intensive } \\
\text { Care Unit (CAM-ICU), assessed by the research team every morning. Missing data on delirium assessment correspond } \\
\text { to one patient in the control group and four in the intervention group. SThe research team assessed pain intensity } \\
\text { every morning at rest by use of the Behavioral Pain Scale (BPS) in patients unable to communicate, or by use of a } \\
\text { visually enlarged 0-10 Numeric Rating Scale (NRS) in patients able to communicate. Severe pain was defined by a BPS } \\
\text { greater than } 5 \text { or an NRS score greater than } 6 \text {. Moderate pain was defined by a BPS of } 4-5 \text { or an NRS of } 4-6 \text {, according } \\
\text { to usual definitions. }{ }^{28} \text { Missing data on pain assessment correspond to one patient in the control group. } 9 \text { Comparisons } \\
\text { between groups were made by use of the log-rank test, with the variable being truncated at day } 28 \text {. }\end{array}$} \\
\hline
\end{tabular}

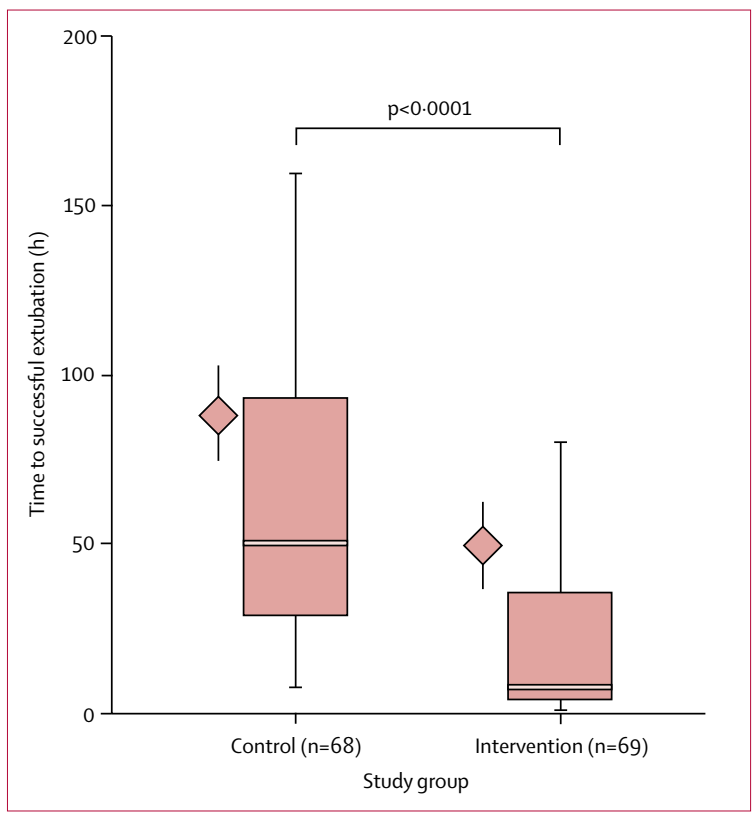

Figure 2: Primary outcome measure, according to study group

Median time between randomisation and successful extubation (the primary outcome) was significantly lower in the intervention group than in the control group: $8 \mathrm{~h}$ (IQR 4-36) versus $50 \mathrm{~h}$ (29-93); mean times were $50 \mathrm{~h}$ (SD 13) versus $89 \mathrm{~h}$ (14), W=966.5 (Mann-Whitney's test), $\mathrm{p}<0.0001$. For the box and whisker plots, the horizontal double bar indicates the median, the upper and lower limits of the boxes the IQR, and the ends of the whiskers the $95 \% \mathrm{Cl}$. The diamonds indicate the means with their bars indicating the standard error.

Guidelines for Management of Severe Sepsis and Septic Shock (Surviving Sepsis Campaign) ${ }^{41}$ recommended minimisation of sedation through appropriate protocols, lighter sedation targets, or daily interruption of sedatives. 


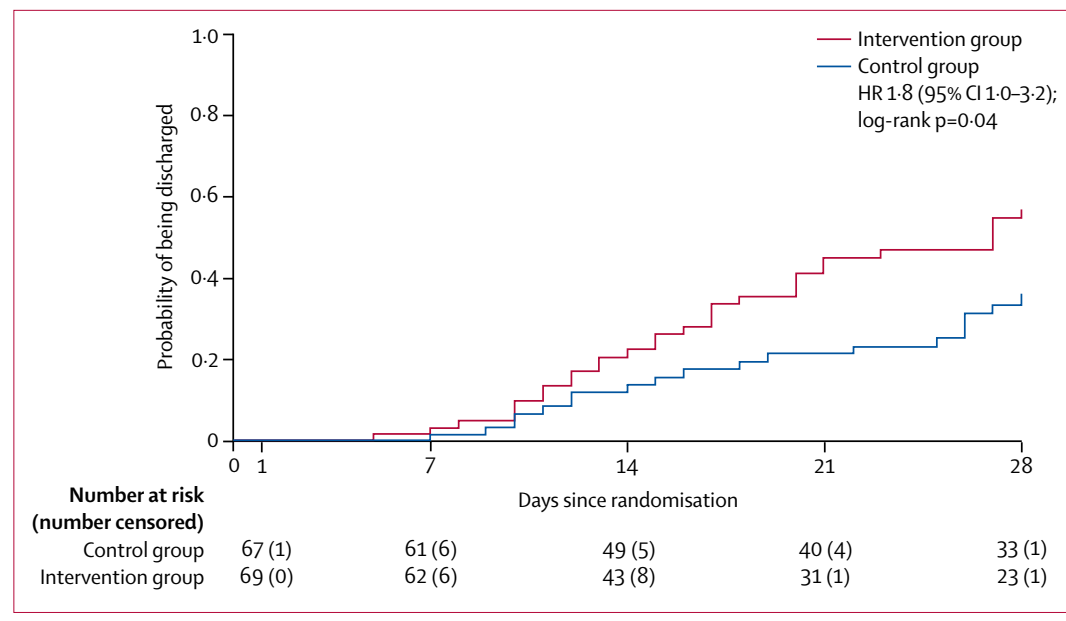

Figure 3: Kaplan-Meier estimates for the probability of being discharged from the hospital at day 28 , according to study group

Deceased patients were censored at death. The $p$ value (with $p<0.05$ indicating statistical significance) was identified by the log-rank test for a between-group difference in the probability of discharge. HR=hazard ratio.

\begin{tabular}{|c|c|c|c|}
\hline & $\begin{array}{l}\text { Control group } \\
(n=68)\end{array}$ & $\begin{array}{l}\text { Intervention } \\
\text { group }(n=69)\end{array}$ & p value \\
\hline $\begin{array}{l}\text { Time between randomisation and first } \\
\text { interruption of sedation, } \mathrm{h}\end{array}$ & $33(23-58)$ & $0 \cdot 3(0-1)$ & $<0.0001$ \\
\hline Resumption of sedation & $19(28 \%)$ & $20(29 \%)$ & 0.89 \\
\hline Reason for first sedation resumption & & & 0.84 \\
\hline Patient-ventilator asynchrony & $13 / 19(68 \%)$ & $11 / 20(55 \%)$ & .. \\
\hline Surgical re-intervention & $4 / 19(21 \%)$ & $6 / 20(30 \%)$ & .. \\
\hline Withdrawal of care & $2 / 19(11 \%)$ & $2 / 20(10 \%)$ & .. \\
\hline Invasive procedure & $0 / 19(0 \%)$ & $1 / 20(5 \%)$ & .. \\
\hline $\begin{array}{l}\text { Time between first interruption of sedation } \\
\text { and first resumption of sedation, } \mathrm{h}\end{array}$ & $62(28-180)$ & $37(3-96)$ & $0 \cdot 10$ \\
\hline Total duration of sedation, $\mathrm{h}$ & $43(26-67)$ & $1(0-6)$ & $<0.0001$ \\
\hline RASS level during continuous sedation* & $-2 \cdot 9(-3 \cdot 8$ to $-2 \cdot 2)$ & $-3 \cdot 5(-4 \cdot 5$ to $-2 \cdot 6)$ & $0 \cdot 17$ \\
\hline Protocol deviation $\dagger$ & $7(10 \%)$ & $1(1 \%)$ & 0.03 \\
\hline \multicolumn{4}{|c|}{$\begin{array}{l}\text { Data are } n(\%) \text { or median (IQR), unless otherwise stated. RASS=Richmond Agitation Sedation Scale. *RASS level during } \\
\text { sedation was calculated among the } 68 \text { patients in the control group, during all sedation periods except when sedation was } \\
\text { deepened during withdrawal of care. In the intervention group, the RASS level during sedation was calculated in the } \\
18 \text { patients for whom sedation was resumed after the initial interruption that was made upon enrolment according to the } \\
\text { study protocol. In the intervention group, the two patients for whom sedation was only resumed during withdrawal of } \\
\text { care were not included in the calculation of RASS level during sedation. IIn the control group, a protocol deviation was } \\
\text { declared if sedation was stopped before the daily interruption of sedation planned every morning. In the intervention } \\
\text { group, a protocol deviation was declared if sedation was resumed for more than } 6 \mathrm{~h} \text { in situations other than those allowed } \\
\text { by the protocol (ie, development of a severe acute respiratory distress syndrome defined as a ratio of the partial pressure of } \\
\text { arterial oxygen to the fraction of inspired oxygen of less than } 150 \text { with a positive end-expiratory pressure of } 5 \mathrm{~cm} \text { or more } \\
\text { of water, or the necessity to resume sedation for more than two } 6 \mathrm{~h} \text { periods within } 24 \mathrm{~h} \text { ). }\end{array}$} \\
\hline
\end{tabular}

Our study in postoperative patients with septic shock suggests that sedation should in fact be completely stopped as soon as possible after ICU admission.

In this population of surgical ICU patients, resumption of sedation was often determined by the need for surgical re-intervention $(30 \%)$, but the main reason was patient-ventilator asynchrony. Sedation is intertwined with mechanical ventilation, as has been previously shown for the development of ventilator-induced diaphragmatic dysfunction: for greater control of mechanical ventilation, more sedatives are required and the risk of ventilator-induced diaphragmatic dysfunction is increased. ${ }^{45-47}$ Consequently, while attempting to reduce the use of sedatives in critically ill patients, it also seems paramount that mechanical ventilation be carefully adapted. ${ }^{30,48}$ Another feature of the present study is the protocolisation of pain, agitation, and delirium management in both groups. This protocol is now recommended as the so-called ABCDEF approach. ${ }^{49}$ Our study represents a step forward by showing that an approach involving reduced use of sedatives and major opioids (which are frequently associated with side-effects such as decreased respiratory drive, delayed ventilator weaning, and increased risk of coma and delirium) should be integrated into a comprehensive strategy for the systematic assessment and management of pain, agitation, and delirium (which should be further associated with early mobilisation).

Strengths of this trial include the methods used to minimise bias (centralised randomisation, complete follow-up, and intention-to-treat analyses). The trial protocol was pragmatic, with recommended practices applied by intensive care staff, including nurses and physicians, by use of validated and reliable instruments for sedation, pain, and delirium assessment, thus making it easy to replicate this study. We measured the time between randomisation and the first interruption of sedatives precisely in both groups. The first no-sedation trial by Strøm and colleagues ${ }^{17}$ interrupted sedation within $24 \mathrm{~h}$ after intubation but the time between enrolment and interruption of sedation was not clearly reported. ${ }^{17}$ Patients were enrolled in our study a few hours after admission to the ICU, compared with 2 days after admission in most previous studies that have investigated the effect of different sedation strategies. . $^{3,7,831,36}$ These previous studies were done mostly in medical ICU patients. However, our trial, together with the trial by Strøm and colleagues, raises the question of whether or not sedation could have been stopped earlier, and whether starting an intervention aimed at minimising sedation within 2 days of admission to the ICU would improve outcomes or not. Although sedation was suspected to be associated with delirium in previous observational studies, ${ }^{5,6,50,51}$ our study is the first interventional study that shows a positive effect of a no-sedation strategy in decreasing the incidence of delirium, contrary to the findings of Strøm and colleagues. ${ }^{17}$ This effect might be explained by the different methods used to measure delirium or the different protocols for management of pain and agitation. For example, in our trial we prioritised multimodal analgesia, restricting the use of morphine, whereas in the trial by Strøm and colleagues boluses of 2.5-5.0 mg were used "as needed".

A limitation of our study is that the durations of mechanical ventilation were shorter than expected compared with previous historical observational data on 
which our hypothesis was based, ${ }^{21}$ leading to possible underpowering with a difference in time to extubation between groups of much less than the expected $72 \mathrm{~h}$. This difference could be explained by a positive effect generated by the research protocols and the fact that clinicians were aware that their practices were being monitored (ie, the Hawthorne effect, where a positive psychological effect in team management can be observed in both groups under investigation, with workers being motivated to improve their skills and efficiency)..$^{52}$ Sedation was thus probably reduced in both groups because of stricter application of recommended practices. Daily interruption of the sedatives in both groups was based on criteria selected by French intensive care societies (see the protocol in the appendix). ${ }^{22}$ These criteria were more restrictive than those of previous studies investigating daily interruption of sedation. ${ }^{3,8}$ Another limitation of our study is that masking was not possible. Possible biases inherent to such trials investigating sedation strategies aimed at decreasing the duration of mechanical ventilation included possible delays in interruption of sedation or extubation of patients in the control group. However, crossover practices appeared to occur in the reverse direction after randomisation, from the intervention group to the control group: sedation was interrupted immediately in $10 \%$ of patients in the control group. To minimise iatrogeny in the control group, we also paid strict attention to the sedation protocol. ${ }^{4}$ The RASS level was targeted between -1 and -3 to avoid deep sedation in the control group. The duration of ventilation was much lower in the control group than in previous studies on sedation protocols, ${ }^{4,36}$ as well as in Strøm and colleagues' study. ${ }^{17}$ This difference suggests that the control group received a high standard of care. However, this difference could also be explained by the characteristics of our study population, which was restricted to postoperative patients, mostly with septic shock but without severe acute respiratory failure at baseline. This description accounts for 75\% (140 of 186) of postoperative adult patients admitted to our ICUs with organ dysfunction. In these patients, sedation could be interrupted earlier than in other ICU populations. Further studies are needed to measure the feasibility of our strategy in medical ICU patients and in patients with more severe acute respiratory failure, as well as in patients undergoing surgery other than abdominal surgery. Further studies are also needed in a large number of ICUs with different patient-to-nurse ratios and different organisational cultures and skillsets regarding the management of sedation-ventilation and anaesthesia practices, including monitoring of neuromuscular blockade to avoid any residual paralysis. ${ }^{53}$ However, a patient to caregiver ratio of 1:1 should probably be recommended for an intubated patient at the early phase of the postoperative period, to assure careful management of pain, agitation, patient-ventilator asynchrony, monitoring of neuromuscular blockade, body temperature, and shivering (supported by specific protocols), as well as regular education and training. All these practices were implemented in our ICUs, but monitoring for residual neuromuscular blockade could have been improved in some patients, which is another limitation of this study. Finally, our trial was not powered to assess secondary outcomes since the study population was small. Additionally, in a small population of selected patients we could not rule out possible safety issues, particularly rare safety events.

In summary, our trial provides evidence that a strategy of avoiding continuous sedation as early as possible, in the absence of residual neuromuscular blockade and hypothermia, compared with usual sedation care, resulted in improvements in several important clinical outcomes in critically ill postoperative patients. Given the clinical and economic burden of critical illness, postoperative morbidity, and the substantial number of patients who could benefit from this strategy, increased attention should be given to prevention of postoperative iatrogenic injury potentially induced by unnecessary sedation and mechanical ventilation. Future studies should investigate whether immediate interruption of sedation has similar effects in other populations, such as medical ICU patients and patients with more severe acute respiratory failure. This approach would avoid the unncessary 1-2 days of sedation (a high risk period for oversedation) observed before enrolment in previous studies done in this setting. ${ }^{14-16}$

\section{Contributors}

GC and SJ were on the trial management committee. GC, SJ, J-YL, J-MC, and GM were on the scientific committee. AP was the Trial Monitoring and Research Coordinator. NM was responsible for statistical and data coordination. GC, SJ, and BJ were on the writing committee. The following investigators at participating sites were responsible for screening of eligible patients and obtaining patients' and families' consent to participate, as well as inclusion, randomisation, and implementation of the research protocol: $\mathrm{MCo}, \mathrm{JC}, \mathrm{BJ}, \mathrm{AdJ}, \mathrm{FB}, \mathrm{MCi}$, J-MD, GC, and SJ at Saint Eloi Hospital (Montpellier, France); CR, LM, and J-YL at Caremeau Hospital (Nîmes, France); and J-MC and EF at Estaing Hospital (Clermont-Ferrand, France).

\section{Declaration of interests}

J-MC reports grants, personal fees, and non-financial support from Baxter, during the conduct of the study. LM reports personal fees from Philips Echo, General Electric Echo, Fresenius Kiabi, and Fresenius Medical Care, outside of the submitted work. EF reports personal fees from Baxter, Fresenius Kabi, and General Electric Healthcare; personal fees and non-financial support from Edwards, non-financial support from Drager, and travel reimbursement from Fisher \& Paykel, outside of the submitted work. SJ reports personal fees from Drager, Fisher \& Paykel, Hamilton, Xenios, and Baxter, outside of the submitted work. All other authors declare no competing interests.

\section{Acknowledgments}

This study was supported by a public grant from Délégation à la Recherche Clinique et à l'Innovation du Groupement de Coopération Sanitaire de la Mission d'Enseignement, de Recherche, de Référence et d'Innovation (DRCI-GCS-MERRI) de Montpellier-Nîmes. We thank all patients who participated in the study, families who gave their consent for participation, and the clinical and research staff at all trial sites, 
without whose assistance the SOS-Ventilation study could not have been completed. We also thank Carey M Suehs for her help with English corrections.

\section{References}

1 Meara JG, Leather AJ, Hagander L, et al. Global Surgery 2030: evidence and solutions for achieving health, welfare, and economic development. Lancet 2015; 386: 569-624.

2 Futier E, Constantin IM, Paugam-Burtz C, et al. A trial of intraoperative low-tidal-volume ventilation in abdominal surgery. N Engl J Med 2013; 369: 428-37.

3 Kress JP, Pohlman AS, O'Connor MF, Hall JB. Daily interruption of sedative infusions in critically ill patients undergoing mechanical ventilation. N Engl J Med 2000; 342: 1471-77.

4 Brook AD, Ahrens TS, Schaiff R, et al. Effect of a nursing-implemented sedation protocol on the duration of mechanical ventilation. Crit Care Med 1999; 27: 2609-15.

5 Ouimet S, Kavanagh B, Gottfried S, Skrobik Y. Incidence, risk factors and consequences of ICU delirium. Intensive Care Med 2007; 33: 66-73.

6 Pandharipande PP, Girard TD, Jackson JC, et al. Long-term cognitive impairment after critical illness. N Engl J Med 2013; 369: 1306-16.

7 Barr J, Fraser GL, Puntillo K, et al. Clinical practice guidelines for the management of pain, agitation, and delirium in adult patients in the intensive care unit. Crit Care Med 2013; 41: 278-80.

8 Mehta S, Burry L, Cook D, et al. Daily sedation interruption in mechanically ventilated critically ill patients cared for with a sedation protocol: a randomized controlled trial. JAMA 2012; 308: 1985-92.

9 Mehta S, McCullagh I, Burry L. Current sedation practices: lessons learned from international surveys. Anesthesiol Clin 2011; 29: 607-24.

10 Gill KV, Voils SA, Chenault GA, Brophy GM. Perceived versus actua sedation practices in adult intensive care unit patients receiving mechanical ventilation. Ann Pharmacother 2012; 46: 1331-39.

11 Dodek P, Chanques G, Brown G, et al. Role of organisational structure in implementation of sedation protocols: a comparison of Canadian and French ICUs. BMJ Qual Saf 2012; 21: 715-21.

12 Carrothers KM, Barr J, Spurlock B, Ridgely MS, Damberg CL, Ely EW. Contextual issues influencing implementation and outcomes associated with an integrated approach to managing pain, agitation, and delirium in adult ICUs. Crit Care Med 2013; 41 (suppl 1): S128-35.

13 Leone M, Ragonnet B, Alonso S, et al. Variable compliance with clinical practice guidelines identified in a 1-day audit at 66 French adult intensive care units. Crit Care Med 2012; 40: 3189-95.

14 Shehabi Y, Bellomo R, Reade MC, et al. Early intensive care sedation predicts long-term mortality in ventilated critically ill patients. Am J Respir Crit Care Med 2012; 186: 724-31.

15 Shehabi Y, Chan L, Kadiman S, et al. Sedation depth and long-term mortality in mechanically ventilated critically ill adults: a prospective longitudinal multicentre cohort study. Intensive Care Med 2013; 39: $910-18$.

16 Balzer F, Weiss B, Kumpf O, et al. Early deep sedation is associated with decreased in-hospital and two-year follow-up survival. Crit Care 2015; 19: 197.

17 Strøm T, Martinussen T, Toft P. A protocol of no sedation for critically ill patients receiving mechanical ventilation: a randomised trial. Lancet 2010; 375: 475-80.

18 Ferreira FL, Bota DP, Bross A, Mélot C, Vincent JL. Serial evaluation of the SOFA score to predict outcome in critically ill patients. JAMA 2001; 286: 1754-58.

19 Papazian L, Forel JM, Gacouin A, et al. Neuromuscular blockers in early acute respiratory distress syndrome. $N$ Engl J Med 2010 363: 1107-16

20 Legall J-R, Lemeshow S, Saulnier F. New Simplified Acute Physiology Score (SAPS II) based on a European/North American multicenter study. JAMA 1993; 270: 2957-63.

21 Chanques G, Constantin J, Jung B, et al. Sedation protocols for intubated patients and non invasive ventilation: additional concepts for a non-iatrogenic intensive care. Crit Care 2009; 13 (suppl 1): P399.

22 Sauder P, Andreoletti M, Cambonie G, et al. Sedation and analgesia in intensive care (with the exception of new-born babies). Ann Fr Anesth Reanim 2008; 27: 541-51.
23 Sessler CN, Gosnell MS, Grap MJ, et al. The Richmond Agitation-Sedation Scale: validity and reliability in adult intensive care unit patients. Am J Respir Crit Care Med 2002; 166: 1338-44.

24 Ely EW, Truman B, Shintani A, et al. Monitoring sedation status over time in ICU patients: reliability and validity of the Richmond Agitation-Sedation Scale (RASS). JAMA 2003; 289: 2983-91.

25 Payen JF, Bru O, Bosson JL, et al. Assessing pain in critically ill sedated patients by using a behavioral pain scale. Crit Care Med 2001; 29: 2258-63.

26 Chanques G, Viel E, Constantin JM, et al. The measurement of pain in intensive care unit: comparison of 5 self-report intensity scales. Pain 2010; 151: 711-21.

27 Chanques G, Jaber S, Barbotte E, et al. Impact of systematic evaluation of pain and agitation in an intensive care unit. Crit Care Med 2006; 34: 1691-99.

28 de Jong A, Molinari N, de Lattre S, et al. Decreasing severe pain and serious adverse events while moving intensive care unit patients: a prospective interventional study (the NURSE-DO project) Crit Care 2013; 17: R74.

29 The Acute Respiratory Distress Syndrome Network. Ventilation with lower tidal volumes as compared with traditional tidal volumes for acute lung injury and the acute respiratory distress syndrome. N Engl J Med 2000; 342: 1301-08.

30 Chanques G, Kress JP, Pohlman A, et al. Impact of ventilator adjustment and sedation-analgesia practices on severe asynchrony in patients ventilated in assist-control mode. Crit Care Med 2013 41: 2177-87.

31 Girard TD, Kress JP, Fuchs BD, et al. Efficacy and safety of a paired sedation and ventilator weaning protocol for mechanically ventilated patients in intensive care (Awakening and Breathing Controlled trial): a randomised controlled trial. Lancet 2008; 371: 126-34.

32 Boles JM, Bion J, Connors A, et al. Weaning from mechanical ventilation. Eur Respir J 2007; 29: 1033-56.

33 Chanques G, Garnier O, Carr J, et al. The CAM-ICU has now a French "official" version. The translation process of the 2014 updated Complete Training Manual of the Confusion Assessment Method for the Intensive Care Unit in French (CAM-ICU.fr) Anaesth Crit Care Pain Med 2017; published online March 29. DOI:10.1016/j.accpm.2017.02.003.

34 Dalton JE, Kurz A, Turan A, Mascha EJ, Sessler DI, Saager L. Development and validation of a risk quantification index for 30-day postoperative mortality and morbidity in noncardiac surgical patients. Anesthesiology 2011; 114: 1336-44.

35 Shin CH, Long DR, McLean D, et al. Effects of intraoperative fluid management on postoperative outcomes: a hospital registry study. Ann Surg 2017; published online March 10. DOI:10.1097/ SLA.0000000000002220.

36 Minhas MA, Velasquez AG, Kaul A, Salinas PD, Celi LA. Effect of protocolized sedation on clinical outcomes in mechanically ventilated intensive care unit patients: a systematic review and meta-analysis of randomized controlled trials. Mayo Clin Proc 2015; 90: 613-23.

37 Schaller SJ, Anstey M, Blobner M, et al. Early, goal-directed mobilisation in the surgical intensive care unit: a randomised controlled trial. Lancet 2016; 388: 1377-88.

38 Barie PS, Hydo LJ, Eachempati SR. Longitudinal outcomes of intra-abdominal infection complicated by critical illness. Surg Infect 2004; 5: 365-73.

39 De Waele J, Lipman J, Sakr Y, et al. Abdominal infections in the intensive care unit: characteristics, treatment and determinants of outcome. BMC Infect Dis 2014; 14: 420.

40 Moore LJ, McKinley BA, Turner KL, et al. The epidemiology of sepsis in general surgery patients. J Trauma 2011; 70: 672-80.

41 Dellinger RP, Levy MM, Rhodes A, et al. Surviving Sepsis Campaign: international guidelines for management of severe sepsis and septic shock, 2012. Intensive Care Med 2013; 39: 165-228.

42 Singer M, Deutschman CS, Seymour CW, et al. The third international consensus definitions for sepsis and septic shock (Sepsis-3). JAMA 2016; 315: 801-10.

43 Shankar-Hari M, Phillips GS, Levy ML, et al. Developing a new definition and assessing new clinical criteria for septic shock: for the third international consensus definitions for sepsis and septic shock (Sepsis-3). JAMA 2016; 315: 775-87. 
44 Seymour CW, Liu VX, Iwashyna TJ, et al. Assessment of clinical criteria for sepsis: for the third international consensus definitions for sepsis and septic shock (Sepsis-3). JAMA 2016; 315: 762-74.

45 Jaber S, Petrof BJ, Jung B, et al. Rapidly progressive diaphragmatic weakness and injury during mechanical ventilation in humans. Am J Respir Crit Care Med 2011; 183: 364-71.

46 Jung B, Constantin JM, Rossel N, et al. Adaptive support ventilation prevents ventilator-induced diaphragmatic dysfunction in piglet: an in vivo and in vitro study. Anesthesiology 2010; 112: 1435-43.

47 Levine S, Nguyen T, Taylor N, et al. Rapid disuse atrophy of diaphragm fibers in mechanically ventilated humans. $N$ Engl J Med 2008; 358: $1327-35$.

48 Jaber S, Lescot T, Futier E, et al. Effect of noninvasive ventilation on tracheal reintubation among patients with hypoxemic respiratory failure following abdominal surgery: a randomized clinical trial. JAMA 2016; 315: 1345-53.
49 Ely EW. The ABCDEF bundle: science and philosophy of how ICU liberation serves patients and families. Crit Care Med 2017; 45: 321-30.

50 Ely EW, Girard TD, Shintani AK, et al. Apolipoprotein E4 polymorphism as a genetic predisposition to delirium in critically ill patients. Crit Care Med 2007; 35: 112-17.

51 Jaber S, Chanques G, Altairac C, et al. A prospective study of agitation in a medical-surgical ICU: incidence, risk factors, and outcomes. Chest 2005; 128: 2749-57.

52 Wickstrom G, Bendix T. The "Hawthorne effect" - what did the original Hawthorne studies actually show? Scand J Work Environ Health 2000; 26: 363-67.

53 Grosse-Sundrup M, Henneman JP, Sandberg WS, et al. Intermediate acting non-depolarizing neuromuscular blocking agents and risk of postoperative respiratory complications: prospective propensity score matched cohort study. BMJ 2012; 345: e6329. 